\title{
Hepatic portal venous flow in patients undergoing tricuspid valve surgery
}

\author{
S F Purkiss, S Fort, T R Graham, P G Mills, C T Lewis
}

\begin{abstract}
Objective-To determine the changes of flow in the hepatic artery and portal vein of patients undergoing tricuspid valve repair together with mitral valve replacement.
\end{abstract}

Design-Non-randomised parallel group study.

Setting-Tertiary referral centre.

Patients-18 patients with mitral valve disease with or without tricuspid regurgitation (age range 46-73; six men) and six patients with coronary artery disease and normal left ventricular function (women age range 39-78; four men).

Interventions-Mitral valve replacement with or without modified De Vega repair of the tricuspid valve or coronary artery bypass surgery.

Main outcome measures-Flow in the hepatic artery and portal vein as measured by duplex Doppler flowmetry before and two to four weeks after operation.

Results-Preoperative hepatic artery flow was less in patients with mitral valve disease than in patients with coronary artery disease $\left(162 \cdot 7(13.1) \mathrm{ml} \cdot \mathrm{min}^{-1} v\right.$ 242.5(6.6) ml.min $\left.{ }^{-1}, p<0.05\right)$. Portal vein perfusion was significantly reduced only in patients with associated tricuspid regurgitation compared with $(844(83)$ ml.min $v$ 1422(64) $\left.\mathrm{ml} . \mathrm{min}^{-1} \mathrm{p}<0.05\right)$. Hepatic perfusion was unaltered after operation in patients undergoing coronary artery bypass surgery $(p>0.05)$. Flow in the hepatic artery and portal vein was improved only in patients undergoing mitral valve replacement with associated tricuspid valve repair $(\mathbf{p}<0.05)$.

Conclusion-Abnormalities of hepatic perfusion can be measured non-invasively in all patients with mitral valve disease but especially in those with associated tricuspid regurgitation needing valve repair. These abnormalities improved two to four weeks after mitral valve surgery and tricuspid valve repair, suggesting that measurement of portal flow might be a useful means of assessing the severity of tricuspid regurgitation.

(Br Heart $\mathcal{F}$ 1994;71:354-357)

Recent developments in pulsed Doppler flowmetry have allowed the development of non-invasive techniques to measure blood flow. Accurate and reproducible measurements of hepatic arterial and portal venous flow have been described. ${ }^{12}$ Patients with mitral valve disease may have impaired hepatic perfusion for two reasons. Firstly, a reduction in forward cardiac output may reduce perfusion in the hepatic artery. ${ }^{3}$ Secondly, pulmonary hypertension and tricuspid regurgitation may impair portal venous perfusion by raising the systemic venous pressure. ${ }^{4}$

We prospectively studied three groups of patients undergoing mitral valve surgery for mitral valve disease with tricuspid regurgitation, mitral stenosis, or mitral regurgitation without tricuspid regurgitation. Preoperative hepatic and portal perfusions were determined by pulsed Doppler flowmetry and compared with control patients undergoing elective coronary artery surgery. The effect of the surgical intervention on hepatic haemodynamics was then assessed two to four weeks after operation.

\section{Patients and methods}

PATIENTS

Eighteen consecutive patients (age range 46-73; six men) undergoing mitral valve replacement and six patients undergoing routine coronary bypass grafting (age range 39-78; four men) without associated procedures were entered into a prospective nonrandomised study.

All patients underwent coronary and left ventricular angiography that showed normal left ventricular function. Hepatic Doppler flowmetry and echocardiography were performed on all patients with mitral valve disease. Additional pressure measurements were taken during the operation on those with suspected tricuspid incompetence. Patients were grouped according to the predominant valve lesion, mitral regurgitation, mitral stenosis, or mitral valve disease with tricuspid regurgitation (table 1 ).

Mitral valves were replaced during cardiopulmonary bypass and cardioplegic arrest. The mitral valve was excised and a biological or mechanical prosthesis was inserted according to the clinical practice of the operating surgeon. A modified De Vega tricuspid annuloplasty was performed through a separate right atrial incision in the five patients undergoing tricuspid annuloplasty.

DUPLEX DOPPLER FLOWMETRY

Hepatic haemodynamics were measured in all patients after they had fasted for six hours. 
Table 1 Details of patients entered into mitral valve haemodynamic study

\begin{tabular}{|c|c|c|c|c|c|c|}
\hline \multirow[b]{2}{*}{ Group (n) } & \multirow{2}{*}{$\begin{array}{l}\text { Age } \\
\text { range }\end{array}$} & \multirow{2}{*}{$\begin{array}{l}\text { Sex }(n) \\
M / F\end{array}$} & \multirow{2}{*}{$\begin{array}{l}\text { Rhythm (n) } \\
\text { SR/AF }\end{array}$} & \multicolumn{3}{|c|}{$L V$ function } \\
\hline & & & & Good & Fair & Poor \\
\hline $\begin{array}{l}\text { CABG } 6 \\
\text { MR } 7 \\
\text { MS } 6 \\
\text { MTR } 5\end{array}$ & $\begin{array}{l}39-78 \\
65-73 \\
55-62 \\
48-65\end{array}$ & $\begin{array}{l}4 / 2 \\
2 / 5 \\
2 / 4 \\
2 / 3\end{array}$ & $\begin{array}{l}6 / 0 \\
4 / 3 \\
4 / 2 \\
3 / 2\end{array}$ & $\begin{array}{l}6 \\
5 \\
6 \\
4\end{array}$ & 1 & \\
\hline
\end{tabular}

LV, left ventricular; SR, sinus rhythm; AF, atrial flutter or fibrillation; CABG, coronary artery bypass grafting; MR, mitral regurgitation; MS, mitral stenosis; MTR, mitral valve disease with tricuspid regurgitation.
The hepatic Doppler perfusion index was calculated from the ratio of the measured flows in the hepatic arterial flow and total hepatic blood flow (sum of hepatic arterial and portal perfusion).

\section{STATISTICAL ANALYSIS}

All data were subjected to the Mann-Whitney $U$ test and $\mathrm{p}<0.05$ was taken to be significant. The results are expressed as means (SEM).

Studies were performed the day before and two to four weeks after operation. The subjects were examined lying supine and measurements of blood velocity were recorded during suspended respiration. The steerable cursor of a duplex Doppler scanner (SSH $160 \mathrm{H}$ Toshiba medical corporation, Europe) was manoeuvred within the vessels of interest (fig 1). In Doppler mode the time averaged velocity over several cardiac cycles was computed.

Cross sectional areas of the hepatic artery were calculated from the diameter on a suspended ultrasound image and the portal vein by outlining the perimeter of the vessels with the tracking ball. A time averaged cross sectional area of the portal vein was calculated from five random images as the cross sectional area was found to vary with the cardiac cycle and respiration. Duplex Doppler blood flow measurements were anatomically constant for the hepatic artery at its proximal segment immediately after the bifurcation of the coeliac trunk and in the portal vein at the free edge of the lesser omentum. Duplex Doppler blood flow was calculated from the time averaged duplex Doppler velocity and the time averaged cross sectional area.

\section{Results}

Mitral valve replacement alone was performed in 13 patients; six had mitral stenosis and seven had mitral regurgitation. Five patients underwent mitral valve replacement and a De Vega annuloplasty for tricuspid regurgitation. Table 2 shows the results of hepatic Doppler flowmetry in all patients before operation.

Basal hepatic arterial flow was significantly reduced in all patients submitted for mitral valve surgery compared with controls (patients undergoing coronary artery bypass surgery) ( $p>0.05$; fig $2(A)$ ).

The portal perfusion of patients with isolated mitral valve disease was not significantly different from controls. Patients with mitral valve disease and tricuspid regurgitation, however, had significantly reduced portal perfusion ( $p>0.01$; fig $2(\mathrm{~B})$ ).

The preoperative hepatic Doppler perfusion index was significantly reduced in all patients undergoing mitral valve surgery compared with controls, except those with mitral regurgitation ( $p>0.05$; fig $2(\mathrm{C})$ ).

Postoperative hepatic arterial and portal venous flow were unchanged in control patients undergoing coronary artery bypass
Figure 1 Technique of duplex Doppler flowmetry. On the right is a cross sectional image of a midline transverse section through the liver with the steerable pulse wave Doppler cursor recording velocity within the proximal hepatic artery. On the left is the Doppler wave form recorded and the velocity.

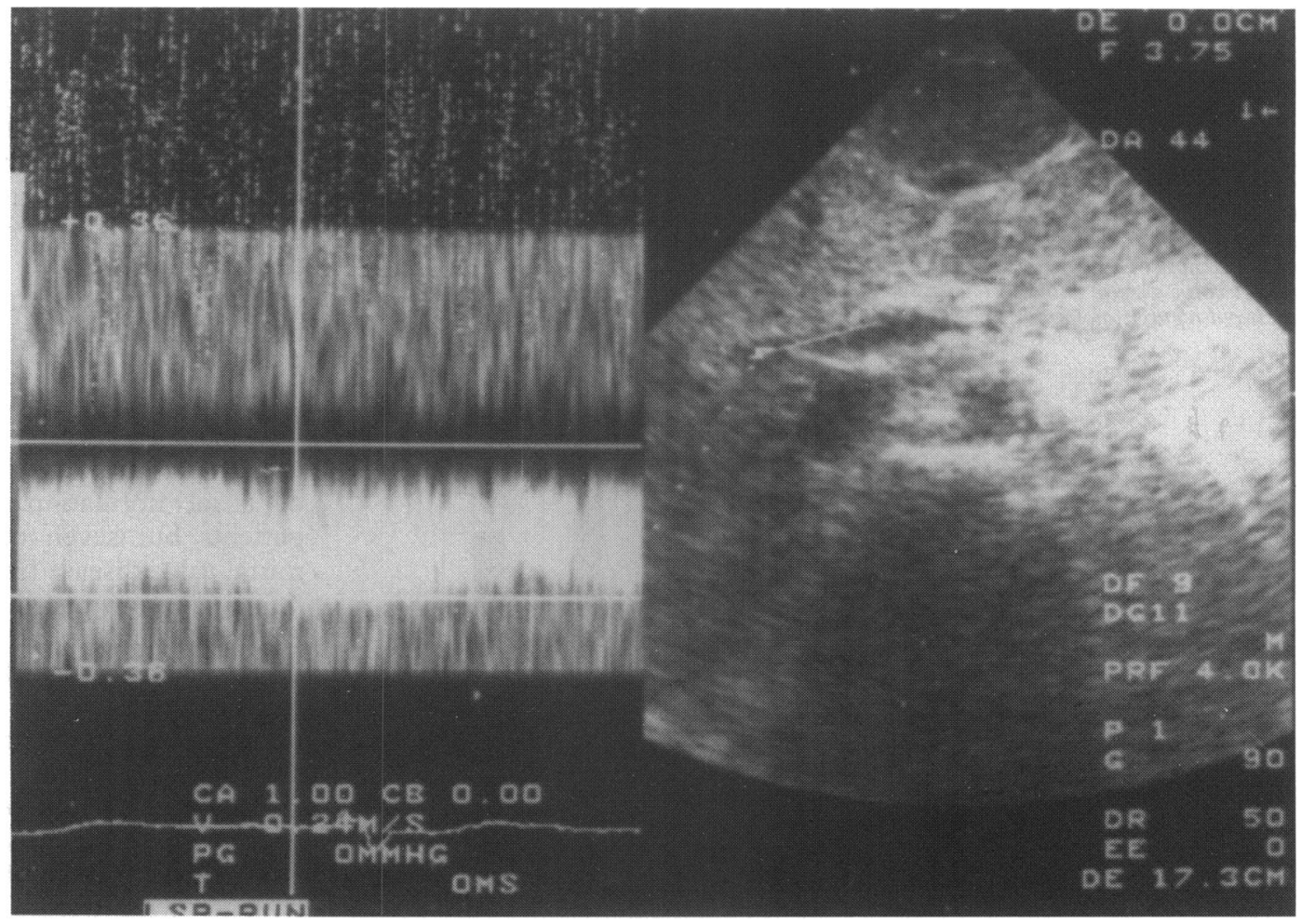


Table 2 Preoperative hepatic haemodynamics of patients (mean (SEM))

\begin{tabular}{|c|c|c|c|c|c|c|c|}
\hline \multirow[b]{2}{*}{ Group } & \multicolumn{3}{|l|}{ Hepatic artery } & \multicolumn{4}{|l|}{ Portal vein } \\
\hline & $\begin{array}{l}\text { Cross sectional } \\
\text { area } \\
\left(\mathrm{mm}^{2}\right)\end{array}$ & $\begin{array}{l}\text { Velocity } \\
\left(\mathrm{cm} \cdot \mathrm{s}^{-1}\right)\end{array}$ & $\begin{array}{l}\text { Flow } \\
\left(\text { ml.min }^{-1}\right)\end{array}$ & $\begin{array}{l}\text { Cross sectional } \\
\text { area } \\
\left(\mathrm{mm}^{2}\right)\end{array}$ & $\begin{array}{l}\text { Velocity } \\
\left(\mathrm{cm} \cdot \mathrm{s}^{-1}\right)\end{array}$ & $\begin{array}{l}\text { Flow } \\
\left(\text { ml.min }^{-1}\right)\end{array}$ & $\begin{array}{l}\text { Doppler } \\
\text { perfusion } \\
\text { index }\end{array}$ \\
\hline $\begin{array}{l}\text { CABG } \\
\text { MR } \\
\text { MS } \\
\text { MTR }\end{array}$ & $\begin{array}{l}16 \cdot 6(1 \cdot 4) \\
17 \cdot 4(1 \cdot 6) \\
17 \cdot 7(0 \cdot 6) \\
14 \cdot 8(1 \cdot 1)\end{array}$ & $\begin{array}{c}14 \cdot 6(1 \cdot 4) \\
11 \cdot 4(1 \cdot 0) \\
10 \cdot 1(0 \cdot 8) \\
7 \cdot 45(0 \cdot 3)\end{array}$ & $\begin{array}{l}242.5(6.6) \\
198.1(16.7) \\
179.8(12.0) \\
110.4(10.8)\end{array}$ & $\begin{array}{l}145 \cdot 1(9 \cdot 8) \\
141 \cdot 7(13 \cdot 6) \\
144 \cdot 0(7 \cdot 0) \\
129 \cdot 2(7 \cdot 9)\end{array}$ & $\begin{array}{r}9 \cdot 8(1 \cdot 0) \\
9 \cdot 7(1 \cdot 2) \\
10 \cdot 1(0.4) \\
6.5(0.6)\end{array}$ & $\begin{array}{c}1422(64) \\
1362(113) \\
1451(81) \\
844(83)\end{array}$ & $\begin{array}{l}0.146(0.03) \\
0.127(0.026) \\
0.110(0.013) \\
0.112(0.027)\end{array}$ \\
\hline
\end{tabular}

Abbreviations as for table 1 .

grafting (fig 2). Hepatic arterial flow was significantly increased compared with preoperative values in patients needing tricuspid valve repair and mitral valve replacement $(110.4$ $\left.(10 \cdot 8) v 205 \cdot 6(7 \cdot 8) \mathrm{ml} \cdot \mathrm{min}^{-1}, \mathrm{p}>0 \cdot 05\right)$.

Portal venous perfusion remained unaltered in all groups except those patients undergoing tricuspid repair as well as mitral valve replacement. Portal perfusion in this group was significantly improved from 844 (83) to 1135 (56) ml.min $\mathrm{m}^{-1}(\mathrm{p}<0.05)$

The doppler perfusion indices in the mitral stenosis and tricuspid regurgitation groups were significantly increased (both $0.15(0.04)$ $\left.\mathrm{ml} . \mathrm{min}^{-1}\right)$ compared with preoperative values $(p<0.05)$. The Doppler perfusion index remained unchanged after surgery in the control patients and those with mitral regurgitation.

\section{Discussion}

Mitral valve disease can have a detrimental effect on liver function as a consequence of abnormal hepatic perfusion. The advanced stage of this complication has been referred to as cardiac cirrhosis and consists of replacement periportal fibrosis as a consequence of tissue hypoxia. ${ }^{5}$ In our study an attempt was

Figure 2 (A) hepatic arterial perfusion, $(B)$ portal perfusion, and (C) Doppler perfusion index (mean (SEM)), before and after surgery. $C A B G$, coronary artery bypass grafting; $M R$, mitral regurgitation; $M S$, mitral stenosis; $M T R$, mitral valve disease with tricuspid regurgitation.

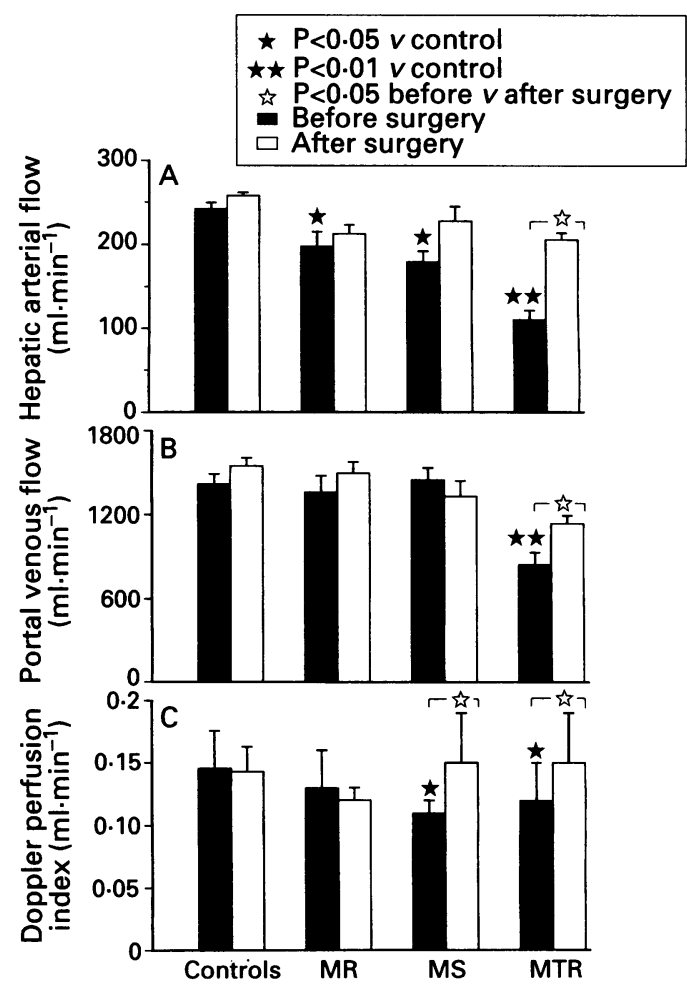

made to quantify the consequences of the haemodynamic abnormalities in patients needing mitral valve replacement. In particular we studied the effect on portal venous flow in the presence or absence of associated tricuspid regurgitation. Patients with normal left ventricular function undergoing coronary artery bypass grafting served as controls.

The technique of hepatic duplex Doppler flowmetry is reproducible and better than other methods of assessing hepatic perfusion because of its ability to measure flow directly within vessels, and its non-invasive nature. ${ }^{12}$ Dynamic hepatic scintigraphy is another method that can assess hepatic haemodynamics. ${ }^{6}$ The technique is unable to measure blood flow directly; as a result a ratio between hepatic arterial and total liver perfusion termed the Doppler perfusion index was introduced. We used this value in our study as it is recognised that perfusion in the hepatic artery and portal vein are proportional to body surface area. ${ }^{1}$ The Doppler perfusion index can therefore be used to express relative hepatic and portal perfusion and can be applied as a comparison between all patients irrespective of body mass and size.

Our study has shown that patients needing mitral valve replacement have profound abnormalities of perfusion of the hepatic artery and portal vein compared with patients with normal left ventricular function undergoing coronary artery bypass grafting. This may be important in many aspects of management of the patients, including treatment involving drugs predominantly metabolised by the liver, and it may also be a useful prediction of those patients likely to develop postoperative jaundice. $^{7}$

After surgery for mitral valve disease many variables of hepatic perfusion were improved Patients undergoing combined mitral and tricuspid surgery had the greatest improvement in hepatic perfusion.

Cardiac output is reduced in the presence of atrial fibrillation. None of the control patients but seven of the 18 patients with mitral valve disease $(39 \%)$ were in atrial fibrillation. The low baseline rates of hepatic arterial flow found in patients with mitral valve disease compared with controls is partly due to the presence of atrial fibrillation. The improvement in hepatic arterial flow in these patients is, however, solely due to the valve replacement as none of these patients reverted to sinus rhythm after surgery.

Portal venous perfusion was reduced in patients with tricuspid regurgitation before 
operation and returned to normal after operation. This may represent the effect of the tricuspid repair on the systemic venous pressure, which affects portal perfusion directly. ${ }^{4}$ The Doppler perfusion index was noted to become more normal in the groups with mitral stenosis and tricuspid regurgitation. Patients who underwent valve replacement for mitral incompetence, however, had a persistently reduced Doppler perfusion index, and this result remains unexplained. The findings that portal and hepatic perfusions and the Doppler perfusion index were improved in patients undergoing tricuspid repair are interesting. Previously the need to perform a tricuspid annuloplasty has relied on clinical features and intraoperative judgment. ${ }^{89}$ The hepatic haemodynamics assessed in our study could be used in conjunction with these methods as an objective guide for the need to repair the tricuspid valve.

In conclusion, duplex Doppler hepatic flowmetry is a useful technique for assessment of hepatic haemodynamics. Before surgery patients with mitral valve disease have abnormal hepatic arterial and portal perfusion. Those patients with tricuspid regurgitation as well as mitral valve disease have the greatest derangement of hepatic blood flow. The abnormalities of hepatic blood flow were sig- nificantly improved after mitral valve replacement. Tricuspid annuloplasty was effective in reversing the additional abnormalities of hepatic blood flow associated with tricuspid regurgitation. Studies of hepatic portal flow may provide a technique for determining which patients with mitral valve disease need tricuspid as well as mitral valve surgery.

1 Leen E, Goldberg JA, Robertson J, et al. The use of duplex sonography in the detection of colorectal hepatic metastases. Br f Cancer 1991;63:323-5.

2 Carlisle KM, Halliwell M, Read AE, et al. Estimation of total hepatic blood flow by duplex ultrasound. Gut total hepatic

3 Altermayer $\mathrm{P}$, Grundmann $\mathrm{U}$, Ziehmer $\mathrm{M}$, et al. Cardiac output and liver blood flow. Methods Find Exp Clin Pharmacol 1991;13:709-71.

4 Reiss WG, Bauer LA, Horn JR, et al. The effects of oral nifedipine in hepatic blood flow in humans. Clin Pharmacol Ther 1991;50:379-84.

5 Sherlock $S$. The liver in heart failure: relation of anatomical, functional and circulatory changes. Br Heart $\mathcal{f} 1951$; 13:273-93.

6 Nott DM, Grime SJ, Yates J, et al. Changes in the hepatic perfusion index during the development of experimental hepatic tumours. Br f Surg 1989;76:259-63.

7 Chu CM, Chang CH, Laiw YF, et al. Jaundice after open heart surgery: a prospective study. Thorax 1984;39:52-6. 8 Surgery for tricuspid regurgitation [editorial]. Lancet 1988; ii: $1061-2$.

9 Cohen SR, Sell JE, McIntosh CL, et al. Tricuspid regurgitation in patients with acquired, chronic, pure mitral regurgitation. 1. Prevalence, diagnosis, and comparison of pre-operative and haemodynamic features in patients with and without tricuspid regurgitation. If Thorac
Cardiovasc Surg 1987;94:481-7. 